Acta Crystallographica Section E

Structure Reports

Online

ISSN 1600-5368

William T. A. Harrison, ${ }^{a} *$ H. S. Yathirajan, ${ }^{\mathrm{b}}$ B. K. Sarojini, ${ }^{\mathrm{c}}$ B. Narayana $^{d}$ and K. K. Vijaya Raj ${ }^{\mathrm{d}}$

${ }^{a}$ Department of Chemistry, University of Aberdeen, Meston Walk, Aberdeen AB24 3UE, Scotland, 'bepartment of Studies in Chemistry,

University of Mysore, Manasagangotri,

Mysore-570 006, India, ' Department of

Chemistry, P. A. College of Engineering,

Nadupadavu, Mangalore-574 153, India, and

dDepartment of Chemistry, Mangalore

University, Mangalagangotri-574 199, India

Correspondence e-mail:

w.harrison@abdn.ac.uk

\section{Key indicators}

Single-crystal X-ray study

$T=120 \mathrm{~K}$

Mean $\sigma(\mathrm{C}-\mathrm{C})=0.002 \AA$

$R$ factor $=0.024$

$w R$ factor $=0.060$

Data-to-parameter ratio $=17.8$

For details of how these key indicators were automatically derived from the article, see http://journals.iucr.org/e.

\title{
2-Bromo-1-chlorophenyl-3-(4-methoxy- phenyl)prop-2-en-1-one
}

The geometrical parameters for the title compound, $\mathrm{C}_{16} \mathrm{H}_{12} \mathrm{BrClO}_{2}$, are normal. The observed bond lengths and angles imply that there is little electronic conjugation between the two benzene ring systems. An intramolecular $\mathrm{C}-\mathrm{H} \cdots \mathrm{Br}$ interaction may help to establish the molecular conformation. The crystal packing results in a centrosymmetric structure.

\section{Comment}

Many chalcone $\left(\mathrm{C}_{15} \mathrm{H}_{12} \mathrm{O}\right)$ derivatives crystallize as noncentrosymmetric structures and display significant non-linear optical (NLO) properties (Uchida et al., 1998). The title compound, (I), (Fig. 1), was prepared as part of our ongoing studies in this area (Harrison et al., 2005). However, (I) crystallizes in a centrosymmetric space group, thus it has a zero NLO response (Watson et al., 1993).<smiles>COc1ccc(/C=C(\Br)C(=O)c2ccc(Cl)cc2)cc1</smiles>

(I)

The geometrical parameters for (I) are normal (Allen et al., 1987) and consistent with those of other chalcone derivatives (Moorthi et al., 2005; Patil et al., 2006). The molecule of (I) is distinctly twisted about the $\mathrm{C} 4-\mathrm{C} 7$ and $\mathrm{C} 7-\mathrm{C} 8$ bonds (Table 1). This twisting, and the $\mathrm{C} 4-\mathrm{C} 7$ and $\mathrm{C} 7-\mathrm{C} 8$ bond lengths of greater than $1.48 \AA$, imply that there is limited electronic conjugation between the two aromatic ring systems. The dihedral angle between the benzene ring mean planes (C1-C6 and $\mathrm{C} 10-\mathrm{C} 15)$ is $53.35(6)^{\circ}$. C7 and $\mathrm{O} 2$ deviate from the former mean plane by 0.176 (3) and 0.895 (3) $\AA$, respectively. By contrast, the terminal methyl atom $\mathrm{C} 16$ is almost coplanar with the C10-C15 ring [deviation $=0.045$ (4) $\AA$ ] .

A PLATON (Spek, 2003) analysis of (I) indicated a possible intramolecular $\mathrm{C}-\mathrm{H} \cdots \mathrm{Br}$ interaction (Table 2) that might help to maintain near coplanarity between the $\mathrm{C} 8 / \mathrm{C} 9 /$ Br1 fragment and the C10-benzene ring. The predicted (Bondi, 1964) van der Waals contact distance for $\mathrm{H}$ and $\mathrm{Br}$ is $3.05 \AA$. There are no $\pi \cdots \pi$ stacking interactions in the crystal structure of (I).

\section{Experimental}

2,3-Dibromo-1-chlorophenyl-3-(4-methoxyphenyl)-2-propan-1-one $(4.32 \mathrm{~g}, 0.01 \mathrm{~mol})$ was mixed with triethylamine $(5 \mathrm{ml}, 0.05 \mathrm{~mol})$ in toluene $(100 \mathrm{ml})$. The reaction was stirred for $24 \mathrm{hrs}$. and the precipitated triethylamine hydrobromide was removed by filtration.
Received 21 March 2006 Accepted 22 March 2006
(C) 2006 International Union of Crystallography All rights reserved 


\title{
supporting information
}

Acta Cryst. (2006). E62, o1578-o1579 [https://doi.org/10.1107/S1600536806010464]

\section{2-Bromo-1-chlorophenyl-3-(4-methoxyphenyl)prop-2-en-1-one}

\author{
William T. A. Harrison, H. S. Yathirajan, B. K. Sarojini, B. Narayana and K. K. Vijaya Raj
}

(I)

Crystal data

$\mathrm{C}_{16} \mathrm{H}_{12} \mathrm{BrClO}_{2}$

$M_{r}=351.62$

Monoclinic, $P 2_{1} / c$

Hall symbol: $-\mathrm{P} 2 \mathrm{ybc}$

$a=13.9793(3) \AA$

$b=8.8780(1) \AA$

$c=11.4870(3) \AA$

$\beta=96.7094(10)^{\circ}$

$V=1415.87(5) \AA^{3}$

$Z=4$

\section{Data collection}

Nonius KappaCCD diffractometer

Radiation source: fine-focus sealed tube Graphite monochromator

$\omega$ and $\varphi$ scans

Absorption correction: multi-scan

SADABS (Bruker, 2003)

$T_{\min }=0.266, T_{\max }=0.573$

Refinement

Refinement on $F^{2}$

Least-squares matrix: full

$R\left[F^{2}>2 \sigma\left(F^{2}\right)\right]=0.024$

$w R\left(F^{2}\right)=0.060$

$S=1.04$

3249 reflections

183 parameters

0 restraints

Primary atom site location: structure-invariant direct methods

Secondary atom site location: none
$F(000)=704$

$D_{\mathrm{x}}=1.650 \mathrm{Mg} \mathrm{m}^{-3}$

Mo $K \alpha$ radiation, $\lambda=0.71073 \AA$

Cell parameters from 3426 reflections

$\theta=2.9-27.5^{\circ}$

$\mu=3.09 \mathrm{~mm}^{-1}$

$T=120 \mathrm{~K}$

Block, colourless

$0.55 \times 0.37 \times 0.18 \mathrm{~mm}$

19150 measured reflections

3249 independent reflections

2906 reflections with $I>2 \sigma(I)$

$R_{\text {int }}=0.039$

$\theta_{\max }=27.5^{\circ}, \theta_{\min }=2.9^{\circ}$

$h=-18 \rightarrow 18$

$k=-11 \rightarrow 11$

$l=-14 \rightarrow 14$

Hydrogen site location: inferred from neighbouring sites

$\mathrm{H}$-atom parameters constrained

$w=1 /\left[\sigma^{2}\left(F_{\mathrm{o}}^{2}\right)+(0.0257 P)^{2}+1.1891 P\right]$

where $P=\left(F_{\mathrm{o}}^{2}+2 F_{\mathrm{c}}{ }^{2}\right) / 3$

$(\Delta / \sigma)_{\max }=0.001$

$\Delta \rho_{\max }=0.36 \mathrm{e}^{-3}$

$\Delta \rho_{\text {min }}=-0.58$ e $\AA^{-3}$

Extinction correction: SHELXL, $\mathrm{Fc}^{*}=\mathrm{kFc}\left[1+0.001 \times \mathrm{xc}^{2} \lambda^{3} / \sin (2 \theta)\right]^{-1 / 4}$

Extinction coefficient: 0.0135 (6)

Special details

Geometry. All e.s.d.'s (except the e.s.d. in the dihedral angle between two 1.s. planes) are estimated using the full covariance matrix. The cell e.s.d.'s are taken into account individually in the estimation of e.s.d.'s in distances, angles and torsion angles; correlations between e.s.d.'s in cell parameters are only used when they are defined by crystal symmetry. An approximate (isotropic) treatment of cell e.s.d.'s is used for estimating e.s.d.'s involving 1.s. planes. 
Refinement. Refinement of $F^{2}$ against ALL reflections. The weighted $R$-factor $w R$ and goodness of fit $S$ are based on $F^{2}$, conventional $R$-factors $R$ are based on $F$, with $F$ set to zero for negative $F^{2}$. The threshold expression of $F^{2}>\sigma\left(F^{2}\right)$ is used only for calculating $R$-factors(gt) etc. and is not relevant to the choice of reflections for refinement. $R$-factors based on $F^{2}$ are statistically about twice as large as those based on $F$, and $R$ - factors based on ALL data will be even larger.

Fractional atomic coordinates and isotropic or equivalent isotropic displacement parameters $\left(\hat{A}^{2}\right)$

\begin{tabular}{|c|c|c|c|c|}
\hline & $x$ & $y$ & $z$ & $U_{\text {iso }} * / U_{\text {eq }}$ \\
\hline $\mathrm{C} 1$ & $0.06540(12)$ & $0.5985(2)$ & $0.21192(16)$ & $0.0146(3)$ \\
\hline $\mathrm{C} 2$ & $0.05661(13)$ & $0.6274(2)$ & $0.32927(17)$ & $0.0168(4)$ \\
\hline $\mathrm{H} 2$ & 0.0102 & 0.6973 & 0.3505 & $0.020^{*}$ \\
\hline $\mathrm{C} 3$ & $0.11671(12)$ & $0.5522(2)$ & $0.41443(16)$ & $0.0155(4)$ \\
\hline H3 & 0.1113 & 0.5701 & 0.4949 & $0.019^{*}$ \\
\hline $\mathrm{C} 4$ & $0.18529(12)$ & 0.45017 (19) & $0.38280(15)$ & $0.0121(3)$ \\
\hline $\mathrm{C} 5$ & $0.19221(12)$ & $0.4226(2)$ & $0.26447(15)$ & $0.0133(3)$ \\
\hline H5 & 0.2387 & 0.3531 & 0.2428 & $0.016^{*}$ \\
\hline C6 & $0.13172(12)$ & $0.4960(2)$ & $0.17835(15)$ & $0.0142(3)$ \\
\hline H6 & 0.1357 & 0.4764 & 0.0978 & $0.017^{*}$ \\
\hline $\mathrm{C} 7$ & $0.23978(12)$ & $0.35782(19)$ & $0.47705(15)$ & $0.0126(3)$ \\
\hline $\mathrm{C} 8$ & $0.34100(12)$ & 0.31417 (19) & $0.46523(15)$ & $0.0125(3)$ \\
\hline C9 & $0.39851(12)$ & $0.39414(19)$ & $0.40209(15)$ & $0.0124(3)$ \\
\hline H9 & 0.3665 & 0.4779 & 0.3636 & $0.015^{*}$ \\
\hline $\mathrm{C} 10$ & $0.49874(12)$ & 0.38168 (19) & $0.37913(15)$ & $0.0124(3)$ \\
\hline C11 & $0.52953(12)$ & $0.4872(2)$ & $0.30057(15)$ & $0.0147(3)$ \\
\hline H11 & 0.4856 & 0.5621 & 0.2685 & $0.018^{*}$ \\
\hline $\mathrm{C} 12$ & $0.62195(13)$ & $0.4858(2)$ & $0.26810(15)$ & $0.0163(4)$ \\
\hline H12 & 0.6408 & 0.5586 & 0.2146 & $0.020^{*}$ \\
\hline $\mathrm{C} 13$ & $0.68678(13)$ & $0.3769(2)$ & $0.31473(16)$ & $0.0164(4)$ \\
\hline $\mathrm{C} 14$ & $0.65847(13)$ & $0.2725(2)$ & $0.39495(18)$ & $0.0196(4)$ \\
\hline H14 & 0.7032 & 0.1993 & 0.4280 & $0.024^{*}$ \\
\hline $\mathrm{C} 15$ & $0.56618(13)$ & $0.2744(2)$ & $0.42692(16)$ & $0.0167(4)$ \\
\hline H15 & 0.5482 & 0.2026 & 0.4817 & $0.020^{*}$ \\
\hline $\mathrm{C} 16$ & $0.81160(14)$ & $0.4659(2)$ & $0.20706(19)$ & $0.0240(4)$ \\
\hline H16A & 0.8783 & 0.4420 & 0.1956 & $0.036^{*}$ \\
\hline H16B & 0.8083 & 0.5691 & 0.2367 & $0.036^{*}$ \\
\hline H16C & 0.7706 & 0.4571 & 0.1321 & $0.036^{*}$ \\
\hline $\mathrm{O} 1$ & $0.77892(10)$ & $0.36308(15)$ & $0.28995(13)$ & $0.0228(3)$ \\
\hline $\mathrm{O} 2$ & $0.20035(9)$ & $0.31697(15)$ & $0.56104(11)$ & $0.0184(3)$ \\
\hline $\mathrm{Cl1}$ & -0.00923 & $0.69297(5)$ & $0.10428(4)$ & $0.02141(11)$ \\
\hline $\mathrm{Br} 1$ & $0.382362(13)$ & $0.14483(2)$ & $0.558415(16)$ & $0.01882(8)$ \\
\hline
\end{tabular}

Atomic displacement parameters $\left(\AA^{2}\right)$

\begin{tabular}{lllllll}
\hline & $U^{11}$ & $U^{22}$ & $U^{33}$ & $U^{12}$ & $U^{13}$ & $U^{23}$ \\
\hline C1 & $0.0103(8)$ & $0.0151(8)$ & $0.0181(9)$ & $-0.0012(7)$ & $-0.0002(6)$ & $0.0050(7)$ \\
C2 & $0.0142(8)$ & $0.0156(9)$ & $0.0214(9)$ & $0.0031(7)$ & $0.0053(7)$ & $0.0006(7)$ \\
C3 & $0.0159(9)$ & $0.0162(9)$ & $0.0150(8)$ & $-0.0007(7)$ & $0.0044(7)$ & $-0.0014(7)$ \\
C4 & $0.0087(8)$ & $0.0131(8)$ & $0.0145(8)$ & $-0.0024(6)$ & $0.0015(6)$ & $0.0007(6)$
\end{tabular}




$\begin{array}{lllllll}\text { C5 } & 0.0099(8) & 0.0142(8) & 0.0161(8) & 0.0003(6) & 0.0032(6) & -0.0009(6) \\ \text { C6 } & 0.0122(8) & 0.0174(9) & 0.0130(8) & -0.0016(7) & 0.0012(6) & 0.0005(7) \\ \text { C7 } & 0.0122(8) & 0.0127(8) & 0.0129(8) & -0.0026(6) & 0.0014(6) & -0.0019(6) \\ \text { C8 } & 0.0125(8) & 0.0119(8) & 0.0125(8) & 0.0021(6) & -0.0012(6) & 0.0003(6) \\ \text { C9 } & 0.0126(8) & 0.0114(8) & 0.0127(8) & 0.0002(6) & -0.0011(6) & 0.0005(6) \\ \text { C10 } & 0.0106(8) & 0.0135(8) & 0.0129(8) & -0.0023(6) & 0.0002(6) & -0.0015(6) \\ \text { C11 } & 0.0129(8) & 0.0159(9) & 0.0147(8) & 0.0003(7) & -0.0008(7) & 0.0019(7) \\ \text { C12 } & 0.0152(8) & 0.0186(9) & 0.0153(8) & -0.0025(7) & 0.0029(7) & 0.0016(7) \\ \text { C13 } & 0.0117(8) & 0.0171(9) & 0.0210(9) & -0.0015(7) & 0.0045(7) & -0.0033(7) \\ \text { C14 } & 0.0151(9) & 0.0150(9) & 0.0290(10) & 0.0038(7) & 0.0034(7) & 0.0039(7) \\ \text { C15 } & 0.0144(9) & 0.0146(9) & 0.0214(9) & -0.0008(7) & 0.0032(7) & 0.0034(7) \\ \text { C16 } & 0.0180(9) & 0.0257(10) & 0.0304(11) & -0.0012(8) & 0.0114(8) & 0.0021(8) \\ \text { O1 } & 0.0129(6) & 0.0220(7) & 0.0355(8) & 0.0016(5) & 0.0106(6) & 0.0050(6) \\ \text { O2 } & 0.0164(6) & 0.0233(7) & 0.0161(6) & -0.0004(5) & 0.0053(5) & 0.0043(5) \\ \text { C11 } & 0.0155(2) & 0.0261(2) & 0.0222(2) & 0.00535(18) & 0.00078(17) & 0.00994(18) \\ \text { Br1 } & 0.01765(11) & 0.01849(11) & 0.02112(11) & 0.00434(7) & 0.00564(7) & 0.00930(7)\end{array}$

Geometric parameters $(\AA, \stackrel{\circ}{)})$

\begin{tabular}{llll}
\hline $\mathrm{C} 1-\mathrm{C} 6$ & $1.385(2)$ & $\mathrm{C} 9-\mathrm{H} 9$ & 0.9500 \\
$\mathrm{C} 1-\mathrm{C} 2$ & $1.392(3)$ & $\mathrm{C} 10-\mathrm{C} 11$ & $1.403(2)$ \\
$\mathrm{C} 1-\mathrm{C} 11$ & $1.7375(18)$ & $\mathrm{C} 10-\mathrm{C} 15$ & $1.406(2)$ \\
$\mathrm{C} 2-\mathrm{C} 3$ & $1.384(3)$ & $\mathrm{C} 11-\mathrm{C} 12$ & $1.386(2)$ \\
$\mathrm{C} 2-\mathrm{H} 2$ & 0.9500 & $\mathrm{C} 11-\mathrm{H} 11$ & 0.9500 \\
$\mathrm{C} 3-\mathrm{C} 4$ & $1.398(2)$ & $\mathrm{C} 12-\mathrm{C} 13$ & $1.390(3)$ \\
$\mathrm{C} 3-\mathrm{H} 3$ & 0.9500 & $\mathrm{C} 12-\mathrm{H} 12$ & 0.9500 \\
$\mathrm{C} 4-\mathrm{C} 5$ & $1.395(2)$ & $\mathrm{C} 13-\mathrm{O} 1$ & $1.357(2)$ \\
$\mathrm{C} 4-\mathrm{C} 7$ & $1.494(2)$ & $\mathrm{C} 13-\mathrm{C} 14$ & $1.396(3)$ \\
$\mathrm{C} 5-\mathrm{C} 6$ & $1.387(2)$ & $\mathrm{C} 14-\mathrm{C} 15$ & $1.382(3)$ \\
$\mathrm{C} 5-\mathrm{H} 5$ & 0.9500 & $\mathrm{C} 14-\mathrm{H} 14$ & 0.9500 \\
$\mathrm{C} 6-\mathrm{H} 6$ & 0.9500 & $\mathrm{C} 15-\mathrm{H} 15$ & 0.9500 \\
$\mathrm{C} 7-\mathrm{O} 2$ & $1.221(2)$ & $\mathrm{C} 16-\mathrm{O} 1$ & $1.432(2)$ \\
$\mathrm{C} 7-\mathrm{C} 8$ & $1.488(2)$ & $\mathrm{C} 16-\mathrm{H} 16 \mathrm{~A}$ & 0.9800 \\
$\mathrm{C} 8-\mathrm{C} 9$ & $1.346(2)$ & $\mathrm{C} 16-\mathrm{H} 16 \mathrm{~B}$ & 0.9800 \\
$\mathrm{C} 8-\mathrm{B} 1$ & $1.8963(17)$ & $\mathrm{C} 16-\mathrm{H} 16 \mathrm{C}$ & \\
$\mathrm{C} 9-\mathrm{C} 10$ & $1.460(2)$ & & 112.6 \\
& & & $117.48(15)$ \\
$\mathrm{C} 6-\mathrm{C} 1-\mathrm{C} 2$ & $121.89(17)$ & $\mathrm{C} 10-\mathrm{C} 9-\mathrm{H} 9$ & $116.02(15)$ \\
$\mathrm{C} 6-\mathrm{C} 1-\mathrm{C} 11$ & $118.98(14)$ & $\mathrm{C} 11-\mathrm{C} 10-\mathrm{C} 15$ & $126.49(16)$ \\
$\mathrm{C} 2-\mathrm{C} 1-\mathrm{C} 11$ & $119.13(14)$ & $\mathrm{C} 11-\mathrm{C} 10-\mathrm{C} 9$ & $122.22(17)$ \\
$\mathrm{C} 3-\mathrm{C} 2-\mathrm{C} 1$ & $118.74(16)$ & $\mathrm{C} 15-\mathrm{C} 10-\mathrm{C} 9$ & 118.9 \\
$\mathrm{C} 3-\mathrm{C} 2-\mathrm{H} 2$ & 120.6 & $\mathrm{C} 12-\mathrm{C} 11-\mathrm{C} 10$ & 118.9 \\
$\mathrm{C} 1-\mathrm{C} 2-\mathrm{H} 2$ & 120.6 & $\mathrm{C} 12-\mathrm{C} 11-\mathrm{H} 11$ & $119.26(16)$ \\
$\mathrm{C} 2-\mathrm{C} 3-\mathrm{C} 4$ & $120.46(16)$ & $\mathrm{C} 10-\mathrm{C} 11-\mathrm{H} 11$ & 120.4 \\
$\mathrm{C} 2-\mathrm{C} 3-\mathrm{H} 3$ & 119.8 & $\mathrm{C} 11-\mathrm{C} 12-\mathrm{C} 13$ & $125.05(16)$ \\
$\mathrm{C} 4-\mathrm{C} 3-\mathrm{H} 3$ & 119.8 & $\mathrm{C} 11-\mathrm{C} 12-\mathrm{H} 12$ & $\mathrm{C} 13-\mathrm{C} 12-\mathrm{H} 12$ \\
$\mathrm{C} 5-\mathrm{C} 4-\mathrm{C} 3$ & $119.64(16)$ & $\mathrm{O} 1-\mathrm{C} 13-\mathrm{C} 12$ & \\
$\mathrm{C} 5-\mathrm{C} 4-\mathrm{C} 7$ & $121.48(15)$ & &
\end{tabular}




$$
\begin{aligned}
& \mathrm{C} 3-\mathrm{C} 4-\mathrm{C} 7 \\
& \mathrm{C} 6-\mathrm{C} 5-\mathrm{C} 4 \\
& \mathrm{C} 6-\mathrm{C} 5-\mathrm{H} 5 \\
& \mathrm{C} 4-\mathrm{C} 5-\mathrm{H} 5 \\
& \mathrm{C} 1-\mathrm{C} 6-\mathrm{C} 5 \\
& \mathrm{C} 1-\mathrm{C} 6-\mathrm{H} 6 \\
& \mathrm{C} 5-\mathrm{C} 6-\mathrm{H} 6 \\
& \mathrm{O} 2-\mathrm{C} 7-\mathrm{C} 8 \\
& \mathrm{O} 2-\mathrm{C} 7-\mathrm{C} 4 \\
& \mathrm{C} 8-\mathrm{C} 7-\mathrm{C} 4 \\
& \mathrm{C} 9-\mathrm{C} 8-\mathrm{C} 7 \\
& \mathrm{C} 9-\mathrm{C} 8-\mathrm{Br} 1 \\
& \mathrm{C} 7-\mathrm{C} 8-\mathrm{Br} 1 \\
& \mathrm{C} 8-\mathrm{C} 9-\mathrm{C} 10 \\
& \mathrm{C} 8-\mathrm{C} 9-\mathrm{H} 9 \\
& \text { C6-C1-C2-C3 } \\
& \mathrm{C} 11-\mathrm{C} 1-\mathrm{C} 2-\mathrm{C} 3 \\
& \mathrm{C} 1-\mathrm{C} 2-\mathrm{C} 3-\mathrm{C} 4 \\
& \mathrm{C} 2-\mathrm{C} 3-\mathrm{C} 4-\mathrm{C} 5 \\
& \mathrm{C} 2-\mathrm{C} 3-\mathrm{C} 4-\mathrm{C} 7 \\
& \mathrm{C} 3-\mathrm{C} 4-\mathrm{C} 5-\mathrm{C} 6 \\
& \mathrm{C} 7-\mathrm{C} 4-\mathrm{C} 5-\mathrm{C} 6 \\
& \mathrm{C} 2-\mathrm{C} 1-\mathrm{C} 6-\mathrm{C} 5 \\
& \mathrm{C} 11-\mathrm{C} 1-\mathrm{C} 6-\mathrm{C} 5 \\
& \mathrm{C} 4-\mathrm{C} 5-\mathrm{C} 6-\mathrm{C} 1 \\
& \mathrm{C} 5-\mathrm{C} 4-\mathrm{C} 7-\mathrm{O} 2 \\
& \mathrm{C} 3-\mathrm{C} 4-\mathrm{C} 7-\mathrm{O} 2 \\
& \mathrm{C} 5-\mathrm{C} 4-\mathrm{C} 7-\mathrm{C} 8 \\
& \mathrm{C} 3-\mathrm{C} 4-\mathrm{C} 7-\mathrm{C} 8 \\
& \mathrm{O} 2-\mathrm{C} 7-\mathrm{C} 8-\mathrm{C} 9 \\
& \mathrm{C} 4-\mathrm{C} 7-\mathrm{C} 8-\mathrm{C} 9 \\
& \mathrm{O} 2-\mathrm{C} 7-\mathrm{C} 8-\mathrm{Br} 1 \\
&
\end{aligned}
$$

$118.33(15)$

120.44 (16)

119.8

119.8

118.83 (16)

120.6

120.6

$121.10(16)$

$119.78(15)$

$119.11(14)$

$123.07(16)$

$124.08(13)$

$112.66(12)$

$134.71(16)$

112.6

$0.6(3)$

$-179.65(14)$

$0.4(3)$

$-0.8(3)$

$-172.42(16)$

$0.2(3)$

$171.47(16)$

$-1.3(3)$

$178.99(13)$

$0.9(3)$

-137.69 (17)

33.7 (2)

40.9 (2)

$-147.67(16)$

$-155.64(17)$

$25.8(2)$

$19.6(2)$
$\mathrm{O} 1-\mathrm{C} 13-\mathrm{C} 14$

$\mathrm{C} 12-\mathrm{C} 13-\mathrm{C} 14$

$\mathrm{C} 15-\mathrm{C} 14-\mathrm{C} 13$

C15-C14-H14

$\mathrm{C} 13-\mathrm{C} 14-\mathrm{H} 14$

C14-C15-C10

C14-C15-H15

$\mathrm{C} 10-\mathrm{C} 15-\mathrm{H} 15$

$\mathrm{O} 1-\mathrm{C} 16-\mathrm{H} 16 \mathrm{~A}$

$\mathrm{O} 1-\mathrm{C} 16-\mathrm{H} 16 \mathrm{~B}$

H16A-C16-H16B

$\mathrm{O} 1-\mathrm{C} 16-\mathrm{H} 16 \mathrm{C}$

$\mathrm{H} 16 \mathrm{~A}-\mathrm{C} 16-\mathrm{H} 16 \mathrm{C}$

$\mathrm{H} 16 \mathrm{~B}-\mathrm{C} 16-\mathrm{H} 16 \mathrm{C}$

$\mathrm{C} 13-\mathrm{O} 1-\mathrm{C} 16$

$\mathrm{C} 4-\mathrm{C} 7-\mathrm{C} 8-\mathrm{Br} 1$

$\mathrm{C} 7-\mathrm{C} 8-\mathrm{C} 9-\mathrm{C} 10$

$\mathrm{Br} 1-\mathrm{C} 8-\mathrm{C} 9-\mathrm{C} 10$

$\mathrm{C} 8-\mathrm{C} 9-\mathrm{C} 10-\mathrm{C} 11$

$\mathrm{C} 8-\mathrm{C} 9-\mathrm{C} 10-\mathrm{C} 15$

$\mathrm{C} 15-\mathrm{C} 10-\mathrm{C} 11-\mathrm{C} 12$

C9- $\mathrm{C} 10-\mathrm{C} 11-\mathrm{C} 12$

$\mathrm{C} 10-\mathrm{C} 11-\mathrm{C} 12-\mathrm{C} 13$

$\mathrm{C} 11-\mathrm{C} 12-\mathrm{C} 13-\mathrm{O} 1$

$\mathrm{C} 11-\mathrm{C} 12-\mathrm{C} 13-\mathrm{C} 14$

$\mathrm{O} 1-\mathrm{C} 13-\mathrm{C} 14-\mathrm{C} 15$

$\mathrm{C} 12-\mathrm{C} 13-\mathrm{C} 14-\mathrm{C} 15$

C13-C14-C15-C10

$\mathrm{C} 11-\mathrm{C} 10-\mathrm{C} 15-\mathrm{C} 14$

C9-C10-C15-C14

$\mathrm{C} 12-\mathrm{C} 13-\mathrm{O} 1-\mathrm{C} 16$

$\mathrm{C} 14-\mathrm{C} 13-\mathrm{O} 1-\mathrm{C} 16$
$115.33(16)$

$119.61(16)$

120.84 (17)

119.6

119.6

$120.58(16)$

119.7

119.7

109.5

109.5

109.5

109.5

109.5

109.5

$117.81(15)$

$-159.02(12)$

$177.38(18)$

2.7 (3)

176.46 (19)

$-2.9(3)$

1.4 (3)

-178.08 (16)

-0.1 (3)

$179.83(17)$

$-1.2(3)$

-179.67 (17)

$1.2(3)$

0.0 (3)

$-1.3(3)$

178.08 (18)

$-1.7(3)$

$179.23(17)$

Hydrogen-bond geometry $\left(\AA,{ }^{\circ}\right)$

\begin{tabular}{lllll}
\hline$D-\mathrm{H} \cdots A$ & $D-\mathrm{H}$ & $\mathrm{H} \cdots A$ & $D \cdots A$ & $D-\mathrm{H} \cdots A$ \\
\hline $\mathrm{C} 15-\mathrm{H} 15 \cdots \mathrm{Br} 1$ & 0.95 & 2.62 & $3.3339(18)$ & 132 \\
\hline
\end{tabular}

